\title{
UAV Photogrammetry and 3D Modelling of Complex Architecture for Maintenance Purposes: the Case Study of the Masonry Bridge on the Sele River, Italy
}

\author{
Massimiliano Pepe ${ }^{1 *}$, Domenica Costantino ${ }^{1}$ \\ ${ }^{1}$ Department of Civil, Environmental, Land, Building Engineering and Chemistry (DICATECh) - Politecnico di Bari, 70126 Bari, Italy \\ ${ }^{*}$ Corresponding author, e-mail: massimiliano.pepe@poliba.it
}

Received: 07 May 2020, Accepted: 26 September 2020, Published online: 22 October 2020

\begin{abstract}
The aim of this paper is to identify a suitable methodology to realize, in an easy and quick way, 3D models of complex structures. To achieve this aim, the first step is to build the 3D model of the scene under investigation using photogrammetric modelling. This task was carried out by the use of algorithms based on Structure from Motion (SfM) - Multi View Stereo (MVS) approaches and using camera-generated images supplied in the UAS (Unmanned Aerial system). Once built the 3D point cloud of the structure under investigation, the geometry of each element was reconstructed with 3D profile reconstruction using Rhinoceros software and a few plug-ins developed in this software. Indeed, this paper shows, through a case study of a masonry bridge of special architectural and historic value built in the middle of 1800 s and located in the south of Italy, the potential of the method developed in order to manage a maintenance or restoration project.
\end{abstract}

Keywords

UAV, masonry bridge, Anafi Parrot, photogrammetry, Rhinoceros, Digital Cultural Heritage

\section{Introduction}

With the diffusion of digital images, Computer Vision (CV) began to develop algorithms able to orientate a sequence of images [1-3]. The image orientation techniques traditionally used in photogrammetry integrated with the new approach has allowed obtaining accurate 3D models, taking advantage of CV's high level of automation, without the need to know information "a priori" and, at the same time, to use rigorous geometric and stochastic models developed through traditional photogrammetry. This approach, called Structure from Motion (SfM) solves the issue of camera positioning and scene geometry simultaneously and automatically, using a highly redundant bundle adjustment based on matching features in multiple overlapping, offset images $[4,5]$. In addition, the use of Multi View Stereo (MVS) algorithms allows densifying the point cloud generated by the SfM approach. Recently, the combination of SfM/MVS algorithms and aerial images obtained by UAS (Unmanned Aerial Systems) platform has revolutionized photogrammetric surveying, allowing obtaining 3D models through low-cost, fast and high quality data acquisition and processing [6]. Thanks to the possible low flying height, UAS (also called "drones" or "UAVs- Unmanned Aerial Vehicles") can collect high-resolution images of the topographic surface.

The acquisition by laser scanning on UAV platform allows obtaining morphological information of the terrain even in areas covered by vegetation; however, this type of acquisition is still limited to a small number of unmanned aircraft [7] and, at moment, is still an expensive technology. In addition, the use of the photogrammetry by UAS is really useful in critical scenarios, such as in harsh high-mountain environments where the easy transportability and low cost of the necessary equipment for aerial survey compared to laser scanning techniques represent a great advantage [8]. The use of the SfM/MVS approach has been successfully applied to detect complex structures, both with terrestrial survey and from UAV platform. Bolognesi et al. [9], performed a comparison between traditional aerial photogrammetry and photogrammetry by UAV and discuss their possible integration in the 3D modelling of a complex building (castle). Russo and Manferdini [10] wrote about the integration between 
different image- and range-based survey techniques, applied to survey a complex architectural case study, Santa Maria di Pomposa Abbey, which exemplifies a wide range of characteristics that it is possible to meet in the survey of the Cultural Heritage field. Kouimtzoglou et al. [11] used an image-based method in 3D reconstruction of complex architectures. In particular, the photogrammetric method was applied in order to build the 3D model of the Plaka Bridge, located in the Epirus region in northwest Greece. Hatzopoulos et al. [12] discussed the digital reconstruction of the archaeological site of Delphi in a specific project named Delphi4Delphi. In this project, various surveying technologies, such as UAS, total station, digital camera were used in order to map in 3D the remaining of the complex monument Tholos and the surrounding area in Delphi Greece. Kaimaris et al. [13] discussed the use of UAV photogrammetry and SfM-MVS approaches in order to locate and map the Traces of the Covered Ancient Theater of Amphipolis (Eastern Macedonia, Greece), which is one of the most important archaeological sites of Greece. Pepe et al. [14] discussed the construction of a three-dimensional model involving a photogrammetric method applied to a Roman bridge (called Ponte san Cono) for structural analysis purposes; in this latter study, the building of 3D models using FEM (Finite Element Method), a novel approach that uses Rhinoceros software to 3D reconstruction of the bridge, is discussed. Starting from this study, we suggest an original method for the survey involving UAV and, once the 3D model has been realized, a suitable procedure for the maintenance and computer management of the structure has been identified. In particular, this last task was carried out by decomposing the bridge into the different objects of which the masonry bridge is composed.

As regards the management of semantic information within the AEC (Architecture, Engineering and Construction) industry, Building Information Modelling (BIM) systems have provided an important and simple tool. However, especially in BIM construction of complex civil constructions such as masonry bridges, only few families of libraries are dedicated to their modelling. In this context, not only from a terminological but also conceptual point of view, it is preferable to use the term HBIM (Historic Building Information Modelling) in the case of parametric modelling of existing objects. Therefore, a novel approach in order to manage geometric and semantic information of a civil structure was proposed and described in this paper.

\section{Materials and experimental procedures}

\subsection{Case study: Ponte del Diavolo masonry bridge}

In the Italian territory, there are numerous masonry bridges that were built in the period from the 1700 s to the 1800 s [15]. The masonry bridges, still today, are important connections in the road network of many Italian zones, some of which fall into areas characterized by a high seismic risk. This means that beyond their historical/aesthetic conservation and restoration, their recovery must also focus on preserving their structural aspect. Therefore, a realistic three-dimensional reconstruction allows a precise analysis of the bridge from a structural, functional and conservation point of view.

A bridge of particular interest, included in the Cultural Heritage, is the so-called Ponte del Diavolo, located in southern Italy, where the coordinates of the centre of the Area Of Interest (AOI) are: latitude 40 $29^{\prime} 50.13^{\prime \prime} \mathrm{N}$ and longitude $15^{\circ} 00^{\prime} 54.26^{\prime \prime} \mathrm{E}$ (Fig. 1a). Indeed, considering the inaccessibility of the lower part of the bridge and its height on the river, this type of bridge is very well suited to the needs of our experimentation.

The masonry bridge on the Sele river is an important structure from an engineering and architectural point of view. Since the Roman times, other bridges were built on this river. However, the power of the river destroyed all the structures created up to the end of the year 1872 (Fig. 1(b)), when the two banks of the Sele river were finally joined by a bridge built with a masonry structure with a single large arch (Fig. 1(c)). The choice of a masonry bridge was due to the low funding of the work. The works began in March 1871 and were completed by 1872, with considerable difficulties, due to the floods of the Sele river and the malaria-ridden environment.

The bridge on the Sele river, designed by the engineer Giustino Fiocca, was built using a type of arch called "basket handle" which involves three centers of curvature: two external ones placed on each side of the vertical symmetry axis and a third one on the vertical symmetry axis, so that the intrados of the arch has a smooth curve [16] (see Fig. 2).

\subsection{Method}

To obtain an accurate textured 3D model, it is necessary to identify a suitable workflow, which can be summarized in two main steps:

- Evaluation of Level of Accuracy (LoA) on the testfield and estimation of the internal camera parameters; 


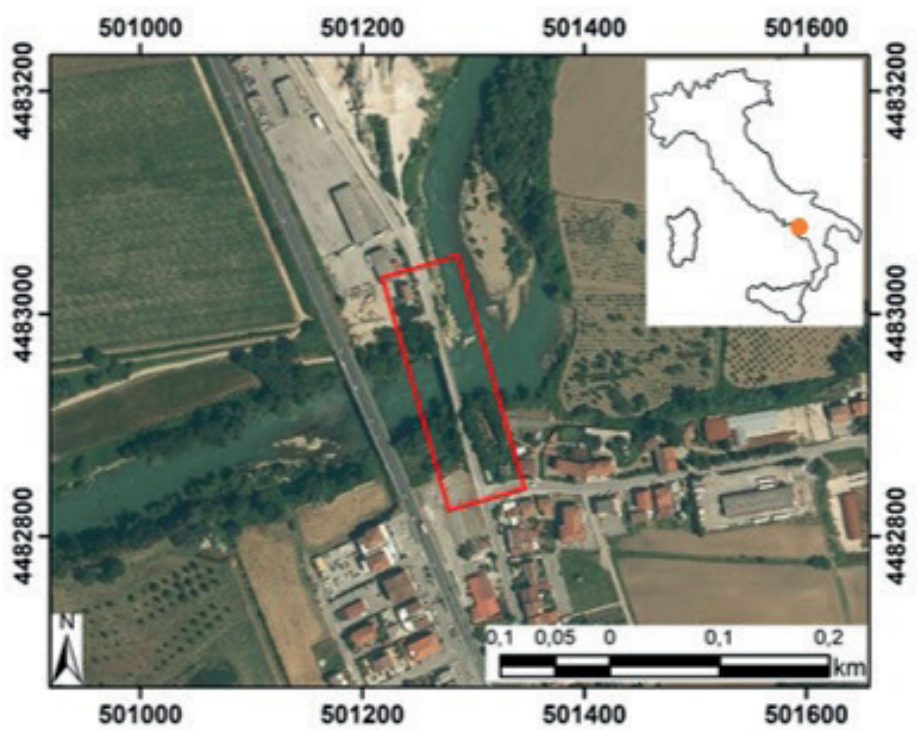

(a)

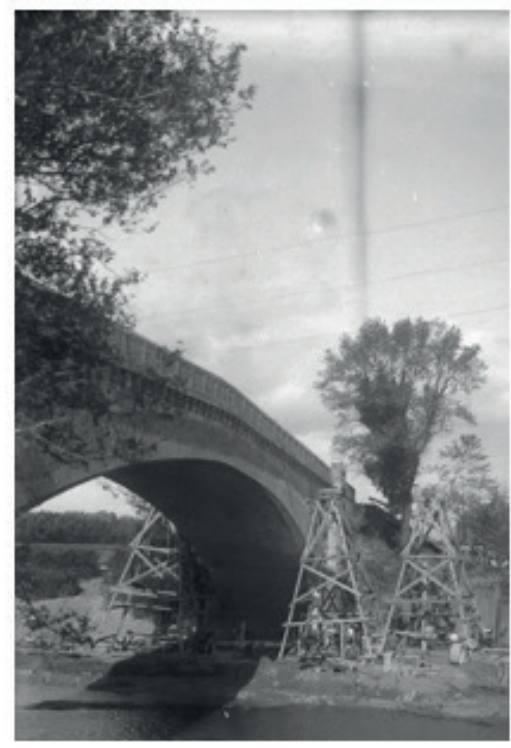

(b)

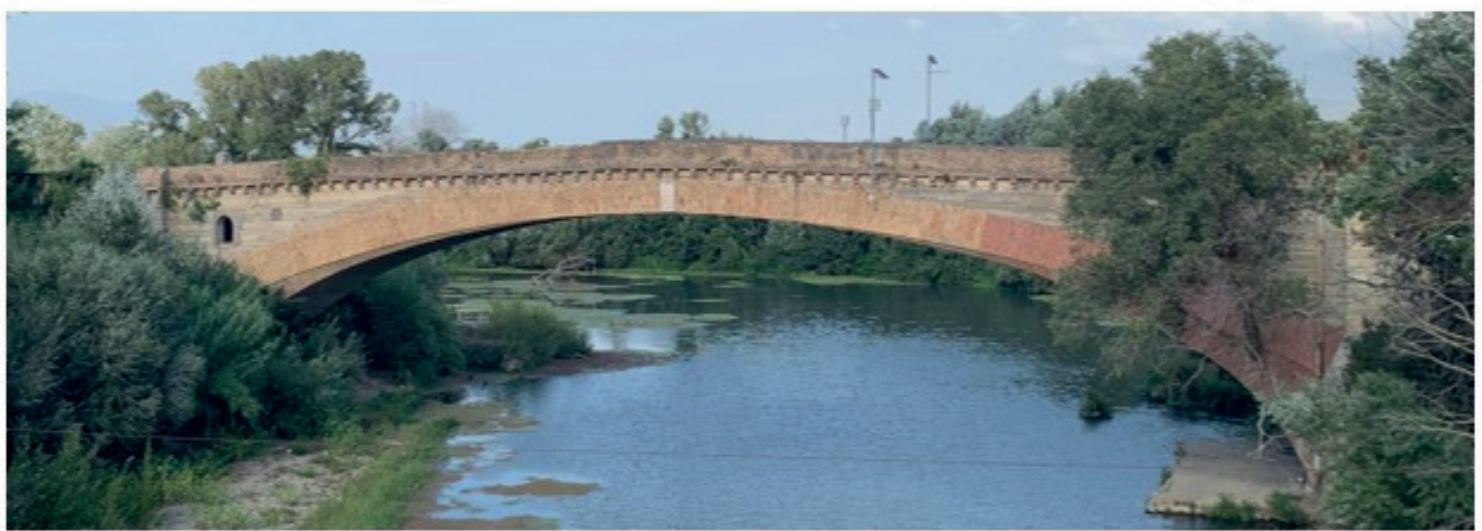

(c)

Fig. 1 Masonry bridge on the Sele river: orthophoto of the area of interest on the Sele river in UTM33N Projection (a), historical image of the Ponte del Diavolo bridge (b) and panoramic image the masonry bridge (c)

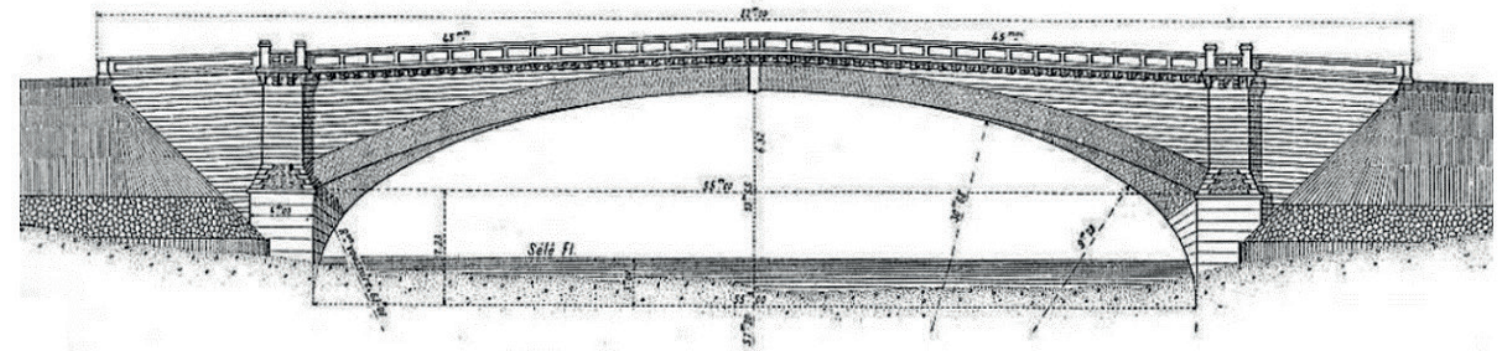

Fig. 2 Façade of the bridge: project by Giustino Fiocca at the end of 1800

- Building of a 3D model on the field using adequate methodology, sensors, an aerial platform and SfM software that are able to reconstruct the geometry of the masonry bridge under investigation.

- 3D modelling of the bridge in a dedicated software in order to build the geometry of the single structure and to link semantic information to these elements.
The first step was the evaluation of the Level of Accuracy of the point clouds generated from a single sensor on 3D test field. Subsequently, once verified that the maximum error on $3 \mathrm{D}$ test-field was acceptable, i.e. that this error was lower than the maximum error compatible with the Scale of Representation (SR) of the masonry bridge, the survey could be performed. 
As regards the second step, it was necessary to take into account the SR and the graphical error drawing (generally established in $0.2 \mathrm{~mm}$ ). In this way, it was possible to calculate the graphical error as the product between the SR and the graphic drawing error. Therefore, once established the tolerable error, the next step consisted in the determination of the Ground Sampling Distance (GSD). As it is known, the GSD of the image can be calculated by the following relation:

$$
G S D=\frac{H}{c} \text { pixel size, }
$$

where:

$H \quad$ is the flight height (or distance from the target to the sensor);

pixel size is the dimension of the sensor and

$c$ is focal length.

In order to obtain a final GSD value lower than the tolerance needed for the project, in general two times the graphical error, a value of half of the originally planned GSD was chosen [17]. In this way, it was possible to design the flight plan on the masonry bridge. Subsequently, to scale the model of the bridge to real dimensions, it was necessary to perform a topographic survey. This latter task was reached using total station instruments featuring the precision required by the project. Once the photogrammetric and topographic survey was performed, the use of software based on SfM-MVS algorithms allowed the construction of a textured 3D model particularly useful for functional restoration.

In order to point out each element of the bridge structure, it is necessary to perform a 3D modelling of the bridge, where each element of the bridge (arch ring, parapets, abutments, key stones, etc.) is geometrically modelled. This task can be accomplished using ComputerAided Design (CAD) and 3D computer graphics software, such as Rhinoceros. In other words, each object in the bridge must be connected to other (simplified or complex) databases. For example, it is possible to create sheets in common data management software that allow exporting in *.pdf files. In fact, with simple hyperlinks it is possible to connect the geometric information produced in Rhinoceros with semantic information. Moreover, in the Rhinoceros environment it is immediately possible to set the type of construction material. The workflow that describes these last steps is reported in Fig. 3.

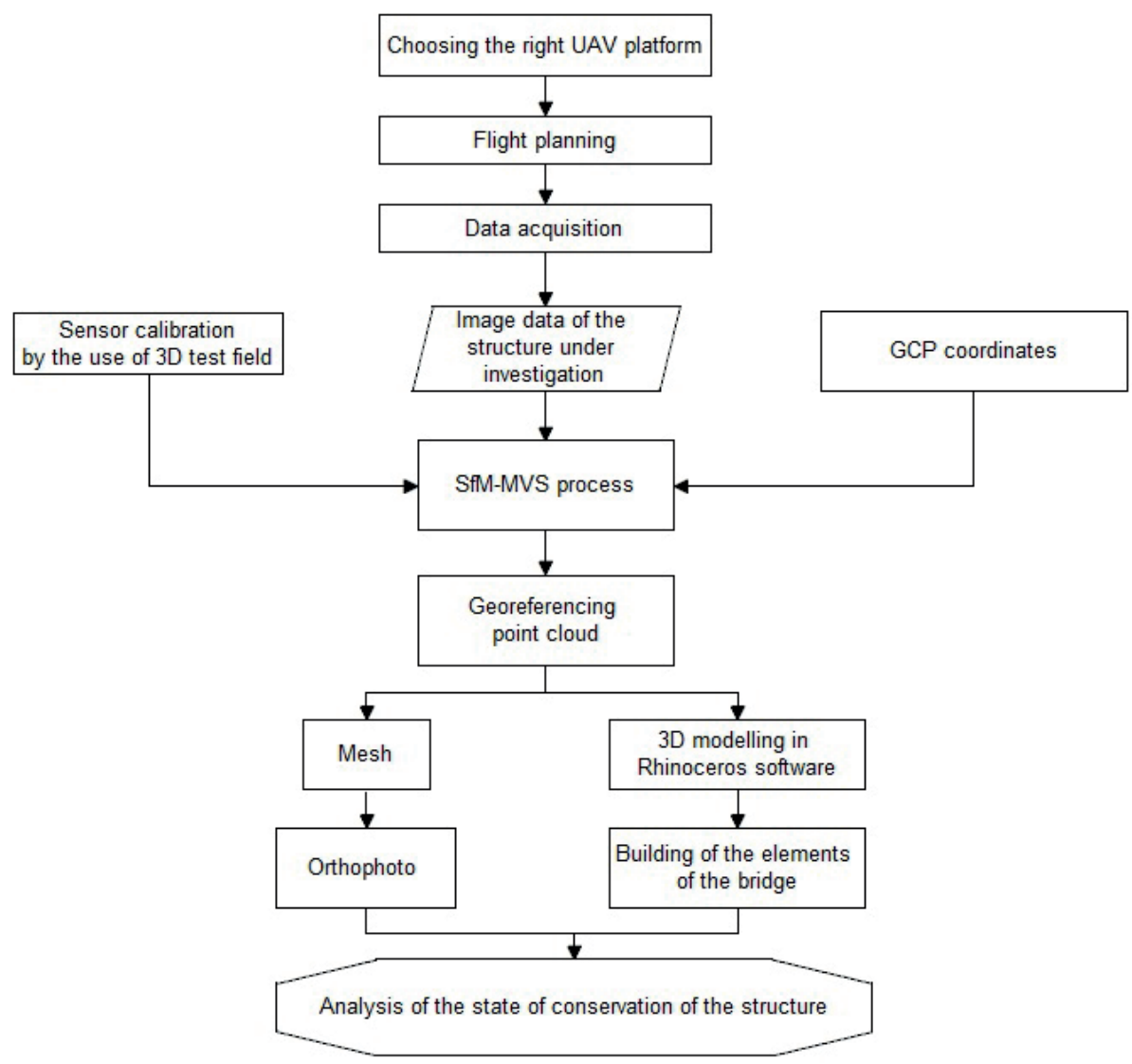

Fig. 3 Workflow of the developed method 


\subsection{Description of the UAS and the software used for the 3D reconstruction of the structure}

The survey of the bridge was carried out using a Parrot Anafi [18], a UAS quadcopter equipped with a Sony Sensor $^{\circledR} 1 / 2.4$ " 21MP $(5344 \times 4016)$ CMOS (complementary metal-oxide semiconductor), which allows obtaining, thanks also to a 3-axis stabilizer, clear and detailed images (Fig. 4(a)). The Anafi allows a rotation of $180^{\circ}$, i.e. a rotation from $-90^{\circ}$ (nadir) to $+90^{\circ}$ (zenith) (Fig. 4(b)).

The Anafi is able to acquire images through a sensor whose pixel size is $0.00134424 \mathrm{~mm}$; the focal length of this camera is $4 \mathrm{~mm}$.

The SfM/MVS software used in the several tests and for the survey of the masonry bridge was Agisoft Metashape because it is a reliable and widely used photo-processing software, as shown in various works present in the literature [19-21]. The procedure that allowed obtaining the 3D model from images can be summarized in the following main steps [22]:

- alignment of the images,

- building the dense cloud,

- building the mesh.

Agisoft Metashape software, after loading the images, performs further operations in the alignment step:

- identifies and matches homologous image points in overlapping images,

- calculates and determines camera position for each image,

- refines camera calibration parameters.

As a result, it generates a sparse point cloud and shows the positions of the cameras. In the second step, based on the estimated camera positions, the software builds a dense point cloud. Lastly, Agisoft Metashape reconstructs a 3D polygon mesh that represents the object, based on the dense point cloud obtained from the calculation of the measurements taken from the surface of the photographed object [23]. Because the elaboration in SfM environment requires high performance from the $\mathrm{PC}$, the

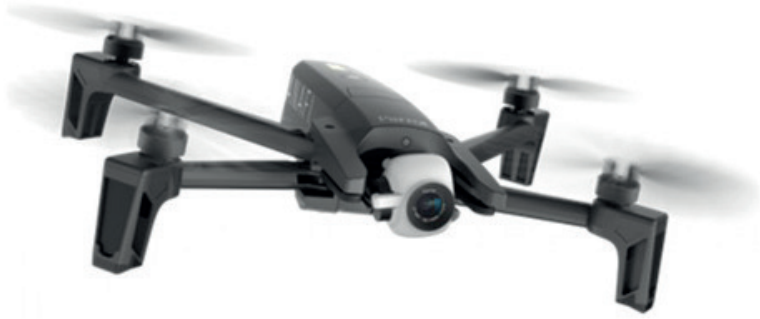

(a)

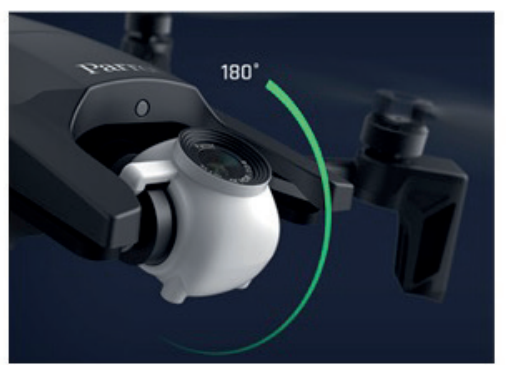

(b)

Fig. 4 Anafi Parrot: UAV platform (a) and zoom on the gimbal where it is shown the possibility to rotate the camera (b) $180^{\circ}$ and, consequently, also in the zenith direction

technical specifications of the PC have an important role. The main feature of the $\mathrm{PC}$ used for the elaboration were: CPU (central processing unit): Intel Core i7 quad-core, LGA 1150 socket, mother board with 4 slot DDR3, RAM: DDR3-1600, $4 \times 4$ GB (16 GB total) and Nvidia GeForce GTX 980 GPU (Graphics Processing Unit).

\subsection{Calibration of the camera supplied with the drone for the construction of the 3D test-field}

The 3D test-field created for this calibration session consisted of 16 circular targets (marker type: 12 bits - coding Agisoft Metashape) positioned at different heights from the ground and spacing included in an area of about $3 \mathrm{~m} \times 3 \mathrm{~m}$.

The coordinates of each target were measured using a Pentax R-322 (N) total station equipped with a laser sensor for readings without prism, whose main technical features (angular and distance accuracy) are reported in the following Table 1.

Table 1 Main features of the Pentax R-322 (N) total station

Measurement range
Accuracy of Electronic Distance
$(1.5 \mathrm{~m}$-Normal range mode:
$90 \mathrm{~m}, /$ Long range mode $200 \mathrm{~m})$


The polar coordinates generated by the total station were transformed into a local Cartesian reference system using a special tool developed in Matlab environment. For each sensor taken into consideration, a block of images with very high overlap and different positions was produced (Fig. 5(b)), thus creating a highly convergent network, as shown in the photogrammetric literature (Fig. 5(a)).

The images generated by the camera supplied with the UAS were processed using Agisoft Metashape in self-calibration mode. The accuracy on the alignment of the images was calculated on 16 targets: 12 were used as Ground Control Points (GCPs) and 4 as Check Points (CPs). The rootmean square (RMSE) evaluated won GCPs was $2 \mathrm{~mm}$ while on CPs it was $3 \mathrm{~mm}$.

In the following Table 2 are reported the values of the internal parameters of the camera where $f$ is the focal length measured in pixels, $c_{x}, c_{y}$ are the principal point coordinates (coordinates of lens optical axis interception with sensor plane in pixels) $b_{1}, b_{2}$ are the affinity and skew (non-orthogonality) transformation coefficients, $k_{1}, k_{2}, k_{3}, k_{4}$ are the radial distortion coefficients, while $p_{1}$ and $p_{2}$ are tangential distortion coefficients.

\subsection{Image acquisition and image processing of the masonry bridge}

Taking into account the Eq. (1) and the features of the cameras, it was possible to establish a relation between the flight height of the drone (i.e. the UAV-object distance) and the GSD. In the case of the sensors tested, the GSD value was $5 \mathrm{~mm}$ by design. This means that the maximum distance between object and camera mounted on the drone was about $4 \mathrm{~m}$.

The upper part of the bridge was surveyed designing the flight plan taking into account the SfM approach and, consequently, choosing suitable camera positions, values of (longitudinal) over-lap (80\%) and (transversal) side-lap [25]. The flight plan was designed using Mission Planner software and created using 8 Flight Lines (FLs) along the longitudinal direction of the bridge, varying the tilt angle in order to cover with high degree of overlap each side of the bridge. In general, the gimbal supplied with the drone allows taking nadir photos, but cannot observe a scene beyond $90^{\circ}$. Instead, in order to overcome this situation, the Anafi drone allows taking photos even in zenith direction (see Appendix A). Therefore, 4 FLs were planned and acquired in manual mode varying

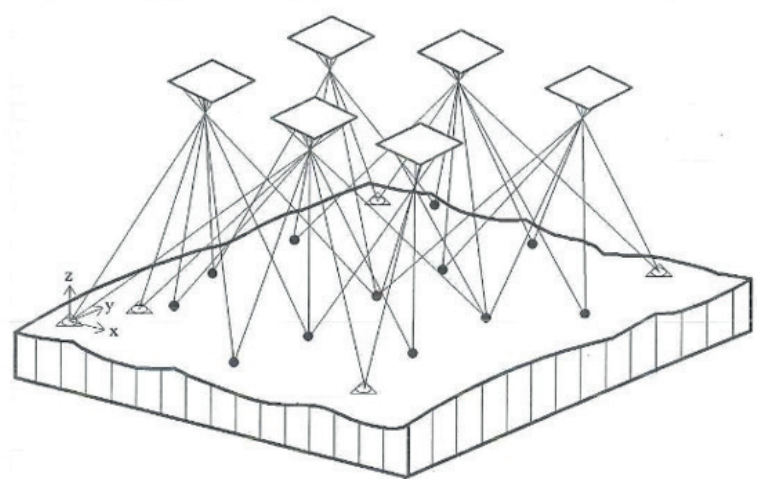

(a)

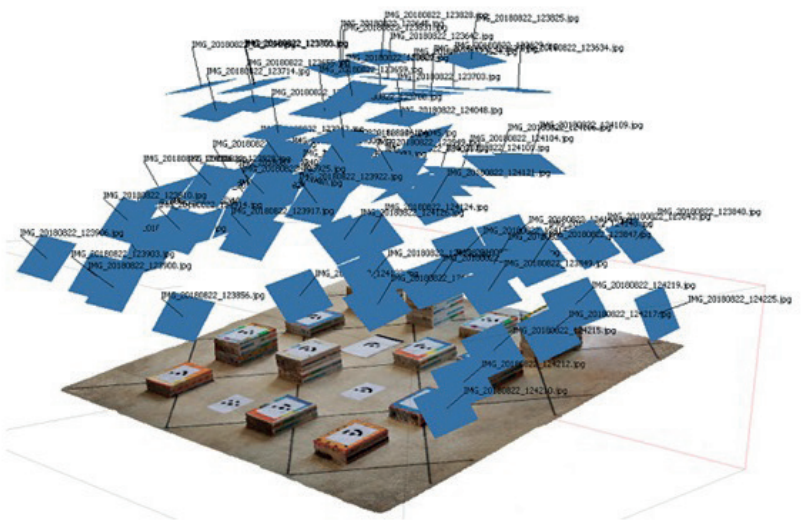

(b)

Fig. 5 Acquisition geometry: (a) sketch of the principle of bundle adjustment (extract from the Kraus book entitled Photogrammetry) [24]; (b) $3 \mathrm{D}$ positions and orientations of the images (blue squares) on test-field

Table 2 Camera calibration parameters

\begin{tabular}{lc}
\hline Parameter & Value \\
\hline$f$ & 2984.013 \\
$c_{x}$ & 0.671585 \\
$c_{y}$ & -0.134793 \\
$k_{1}$ & -0.00341261 \\
$k_{2}$ & 0.0112115 \\
$k_{3}$ & -0.00730694 \\
$b_{1}$ & -0.850948 \\
$b_{2}$ & 0.803138 \\
$p_{1}$ & -0.00120118 \\
$p_{2}$ & -0.00265686 \\
\hline
\end{tabular}

the flight heights in order to obtain the same GSD. In this way, it was possible to observe the intrados of the bridge using a UAV platform.

The UAV used for the experimentation is the Anafi Parrot, a multi-copter rotary wing type that allows achieving a max flight horizontal speed of $15 \mathrm{~m} / \mathrm{s}$ with a max climbing speed of $4 \mathrm{~m} / \mathrm{s}$ while the maximum wind resistance is $50 \mathrm{~km} / \mathrm{h}$. The weight of the vehicle is $320 \mathrm{gr}$. 
As usual, before using an aerial platform in the airspace, it was necessary to check if it was possible to fly on the area of interest. For this reason, it was necessary to plan the flight operations according to the Airspace. The Italian Airspace Structure is divided in 3 FIRs (Flight Information Regions): Milano, Roma and Brindisi.

The area of interest is located in the Brindisi FIR. Using the Brindisi VFR (Visual Flight Rules) map (Scale 1: $500,000)$ and zooming on the area of interest, it is possible to note that the flight zone falls inside the CTR (Controlled Traffic Region), i.e. a volume of controlled airspace which extends from the surface to a specified upper limit, established to protect air traffic operating to and from that airport. In this zone, the Unmanned Aerial Systems Italian Regulation approved by Italian Civil Aviation Authority (ENAC) has provided restrictions on flying over these areas: weight must be less than $25 \mathrm{~kg}$, maximum distance between pilot and UAV must be $200 \mathrm{~m}$ and the maximum flight height must be 70m AGL (Above Ground Level). Therefore, considering the flight planning parameters determined in the previous section, it was possible to fly with UAV also in compliance with aeronautical rules.

In order to carry out the aerial survey, four consecutive sessions were performed in relation to the autonomy of the UAV (batteries last about 25 minutes). Two sessions were used to survey the upper part of the bridge and one to survey the lower part of the bridge.

In the acquisition step, a total amount of 796 images were acquired. The aerial survey was carried out on a morning around 11 a.m. in May. The wind was almost absent. In the acquisition step, close attention was paid in acquiring with uniform light condition. In fact, the survey was carried out with totally cloudy weather condition and, consequently, without shadows. This led to a more pleasant appearance of the bridge model from an archi-tectural and visual point of view and, at same time, avoided strong light contrasts during the acquisition of the bridge substructure, i.e. with image acquisition pointing towards the zenith.

In agreement with Federman et al. [26], the images were first manually inspected and checked to ensure they were not blurry and would be useful in the creation of the model. Because the camera supplied with the Anafi has a large field of view, the images showed a large value of overlap. Consequently, this feature positively improved the image alignment process, as in fact all the images were aligned correctly. Before building the dense cloud, more masks were created on the images. In this way, it was possible to reduce the amount of residual noise on the 3D model. Agisoft
Metashape software offers several options in building dense point clouds (Low, Medium, High, Ultra High): the "Ultra High" setting was used. In this way, it was possible to obtain very realistic structure models, as shown in Fig. 6.

Lastly, the mesh was built from the dense point cloud. To remove small isolated mesh fragments, a special option implemented in the software, called "Select Gradual Selection", allowed deleting the noise in the final model. Table 3 shows the main processing parameters defined (input) and the features in output generated by processing in Agisoft Metashape software.

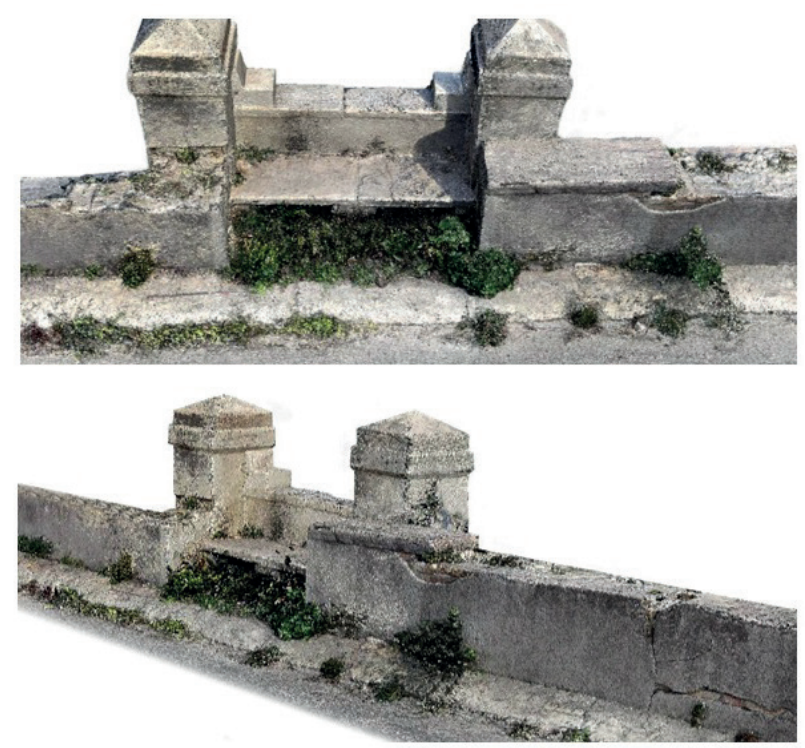

Fig. $63 \mathrm{D}$ point cloud of part of the superstructure of the masonry bridge

Table 3 Summary of input and output parameters

\begin{tabular}{lc}
\hline Features & Numbers \\
\hline No. of images & 768 \\
Key points limit & 40,000 \\
Tie points limit & 12,000 \\
Point clouds & $1,0578,000$ (Ultra High quality) \\
3D Model & $28,722,671$ (faces) \\
\hline
\end{tabular}

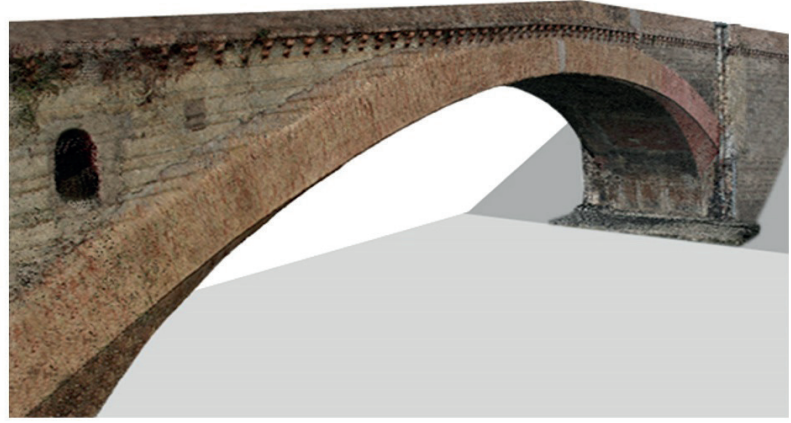

Fig. 7 Textured 3D model of the masonry bridge under investigation 
Therefore, at the end of the processing, the software was able to produce a textured 3D model, as shown in Fig. 7.

The real dimensions of the model were obtained with the use of markers. In addition, the markers were used for different purposes: (i) creation of a coordinate system, (ii) optimization of the alignment of the images, (iii) measurement of distances within the scene. The positions of the markers were defined on the projections of the photos within the software. In this project, a number of 12 cross-coded markers were positioned on the bridge and measured using Pentax N-X 322 total station. The coordinates were obtained using dedicated software that is able to import the data acquired by the total station. The coordinates of the markers were framed in a local coordinate system. By importing the coordinates of the markers into Agisoft Metashape, it was possible to calculate the error in the bundle adjustment step. The RMSE value archived was $9 \mathrm{~mm}$, which was within the expected tolerance of the representation scale, i.e. $10 \mathrm{~mm}$ for 1:50 scale. Once obtained the $3 \mathrm{D}$ model in real dimension, it was possible to generate the orthophoto of the top view and the single façade of the bridge (2D) according to the traditional approach. While the orthophoto of the plan was generated in the reference system created with the markers, for the orthophoto of the bridge façades it was necessary to create a new reference system for each single façade. By defining the origin of the reference system of each single façade, the GCPs were roto-translated. This task was carried out using CAD software and a special tool developed in Matlab environment. Importing the new coordinates of the markers in the Agisoft Metashape project carried out previously, it was possible to build the orthophotos of the two façades of the bridge with a GSD of $0.005 \mathrm{~m}$. The $\mathrm{X}$ axis is parallel to the longitudinal axis of the bridge while the $\mathrm{Z}$ axis is perpendicular to it (vertical axis). In this way, it is possible to obtain a traditional representation of the plan and the facades of the bridge. The architectural layout is shown below (Fig. 8).

\subsection{Building of the geometries of the single elements of the bridge}

The realization of the 3D model and 2D façades provided the basic elements for the restoration and maintenance project of masonry bridges. Now, it is necessary to re-build the mesh putting together each element of the bridge. Indeed, the vegetation on the bridge and especially the need to describe the different components of the structure (pylon, arches, etc.) led to the need to build meshes in a three-dimensional modelling software. In this way, it was possible to construct regular, continuous meshes that, at the same time, represented specific structural objects. Therefore,
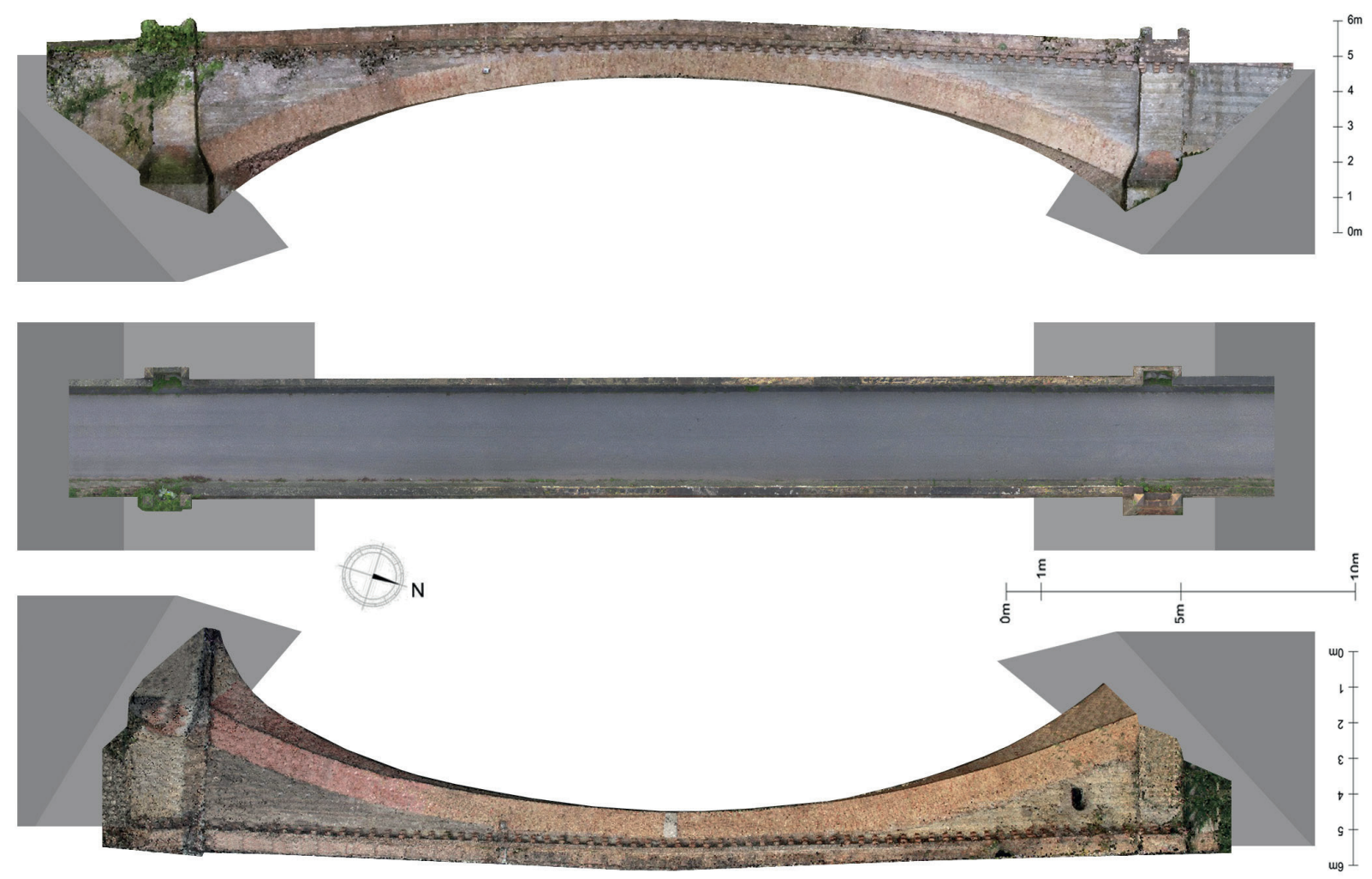

Fig. 8 2D representation of the Ponte del Diavolo masonry bridge 
it is possible to associate to each structural semantic information for the digital management of the masonry bridge. To achieve this aim, the first step was to work on the point cloud within the Rhinoceros 6.0 software [27]. Indeed, from the point cloud, it is possible to build, in Rhinoceros environment, a detailed model of every single element. The construction of the objects was made possible by creating special profiles directly on the point cloud thanks to the use of Arena 4D tool [14]. For example, a vector representation of the point cloud during the construction phase is shown below (Fig. 9). In this way, it was possible to build the objects that make up the bridge. In particular, each object (road, pilaster, arch, etc.) was built on a specific layer, as shown in Fig. 10.

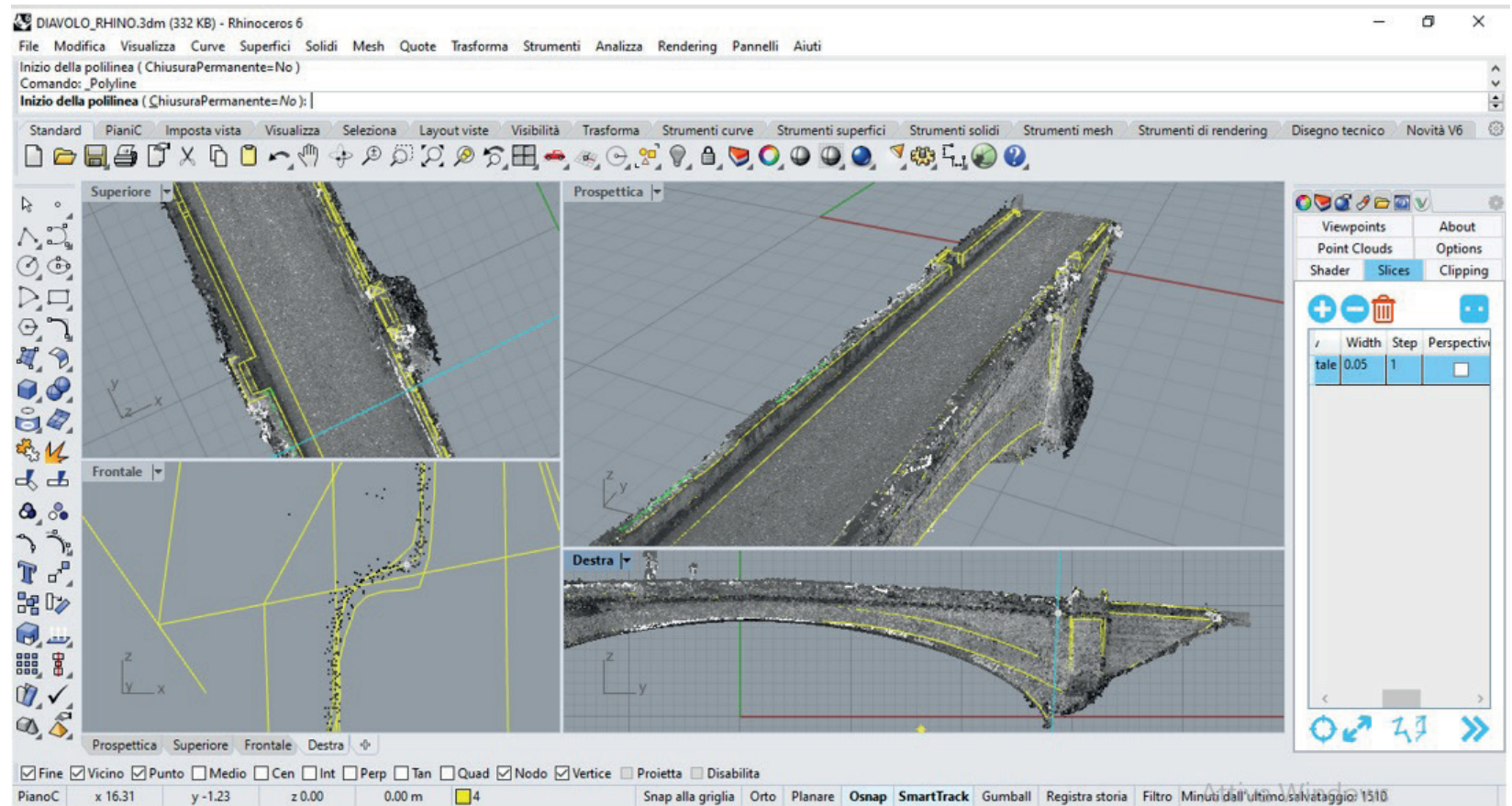

Fig. 9 Modelling in Rhinoceros software and use of the plug-in Arena 4D for the construction of the elements

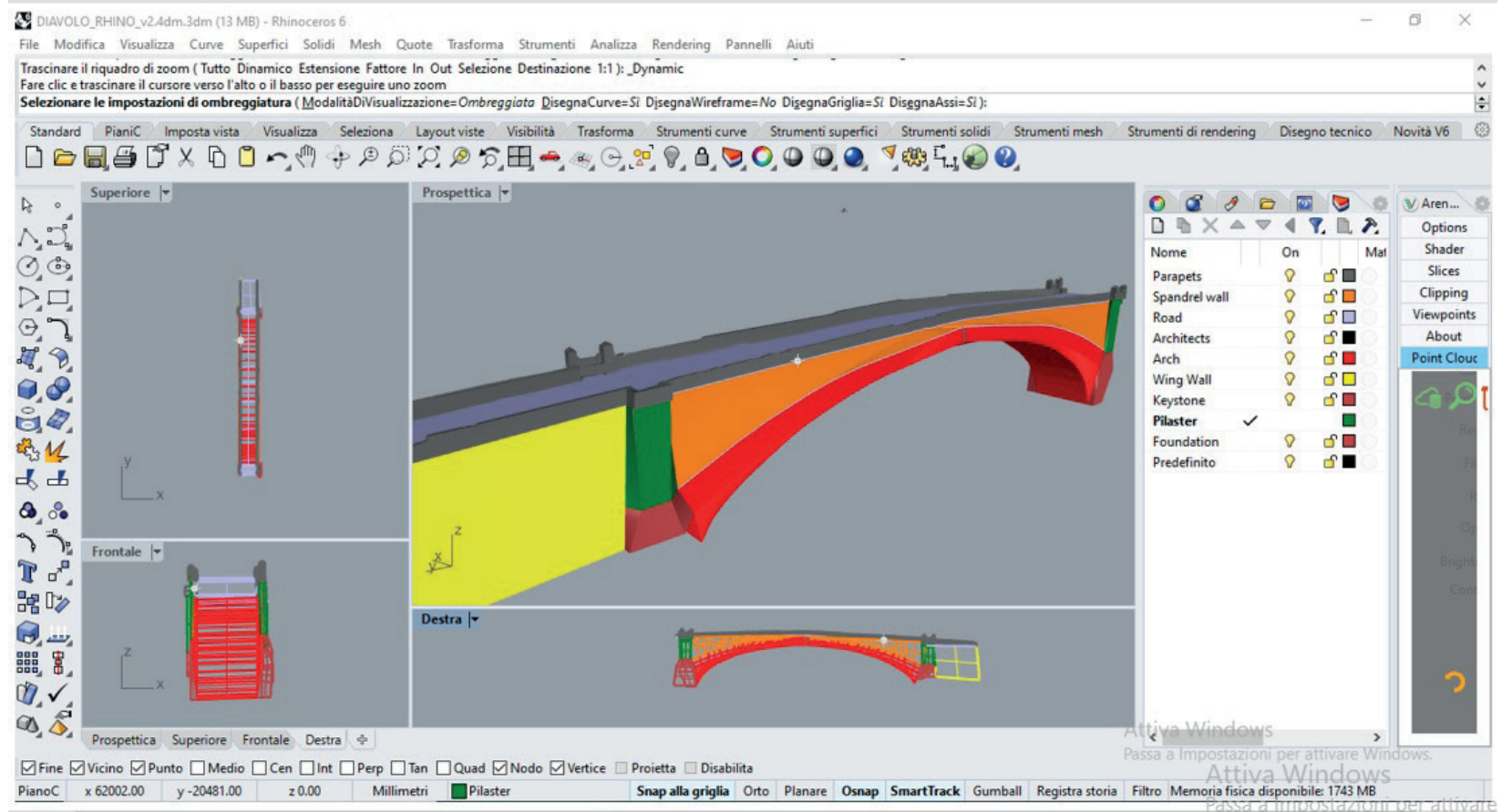

Fig. 10 3D modelling of the masonry bridge in Rhinoceros according different layers 


\subsection{Analysis of the state of degradation}

The reconstruction of the masonry bridge, carried out by photogrammetric survey, shows that the artefact is in a poor state of conservation and the advanced state of deterioration of the superstructure is evident. From the analysis of 2D (plan and façades) and 3D model, it was therefore possible to obtain a great deal of information on the causes of the deterioration, the presence and course of the lesions, the construction techniques, the relationship between the different structures that make up the element and the function of the architectural parts, the form and implementation of the materials. In this way, it was possible to describe the state of health of the bridge and the state of conservation of the characteristic architectural elements of the structure. From a visual analysis of the structure, the bridge does not show any evident cracks; in fact, there are no lesions that can compromise its staticity. However, the state of deterioration and alteration of the structure is evident: some building elements (wall cladding) have collapsed or are seriously damaged; the plaster is detached at various points. In addition, the vegetation has invaded several structure elements creating problems of detachment of materials. The pathologies identified are:

- erosion: abrasive action exerted by atmospheric agents;

- detachment: solutions of discontinuity between the superficial layers of the material that generally prevent them from falling;
- differential degradation: degradation to be put in relation to heterogeneity of composition of the material;

- biological patina: soft and homogeneous thin layer, adherent to the surface and of evident biological nature, of variable color mostly green;

- gap-loss: fall and loss of parts;

- vegetation: presence of vegetable organisms;

- exfoliation: lamellar detachment of the surface layers of the material;

- stain: alteration of accidental pigmentation on the surface and presence of material extraneous to the substrate;

- efflorescence: formation of whitish colored substances of crystalline or filamentous appearance;

- runoff: action of water flowing on the stone element, removing some of its components;

- disintegration: advanced stage loss of cohesion, characterized by the detachment of granules and crystals under minimal mechanical stress.

The activities listed above are part of a structure maintenance and restoration activity to be managed within the modelling software. In fact, Rhinoceros software contains, inside the property of each object, a hyperlink field, to which it is possible to link information-files via ftp, web, files, etc. For example, it is possible to link the parapet object to a specific sheet generated in another environment and available in *.pdf file, as shown below (Fig. 11).

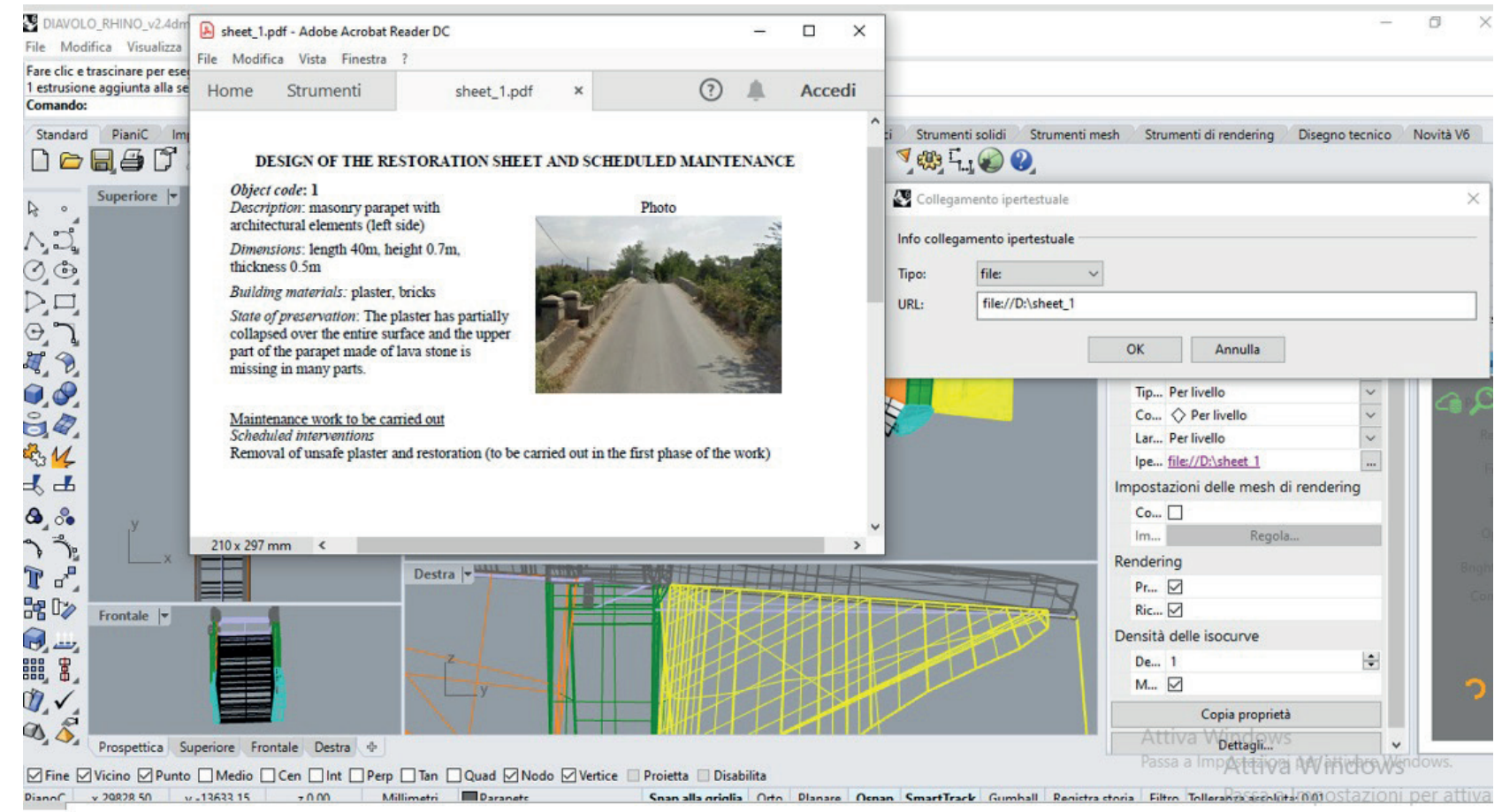

Fig. 11 Link between object and maintenance information board by hyperlink 


\section{Conclusions}

In this paper, it was shown how it was possible to build a 3D model of a complex structure, such as masonry bridges that cannot be surveyed by terrestrial survey. Indeed, in the case of the bridge taken into consideration, the natural obstacles and the large dimension of its span did not allow observing the intrados of the structure using traditional terrestrial survey techniques, such as the Terrestrial Laser Scanner. To overcome this issue, an appropriate technological device and suitable methodology based on the SfMMVS approaches were adopted. The technological solution mounted on UAV allowed obtaining images according to different camera positions. In this way, it was possible to acquire every part of the bridge using only images acquired through a UAV aerial platform, without the need to perform a terrestrial survey: zenith photos were used to cover the intrados of the structure while nadir and oblique photos covered the remaining part of the structure. The suitable photogrammetric pipeline allowed obtaining an accurate 3D model of the masonry bridge (including texture) of high quality.

\section{References}

[1] Lowe, D. G. "Distinctive Image Features from Scale-Invariant Keypoints", International Journal of Computer Vision, 60(2), pp. 91-110, 2004.

https://doi.org/10.1023/B:VISI.0000029664.99615.94

[2] McGlone, J. C., Mikhail, E. M., Bethel, J. S., Mullen, R. "Manual of photogrammetry", American Society for Photogrammetry and Remote Sensing, Bethesda, MD, USA 2004.

[3] Brown, M., Lowe, D. G. "Unsupervised 3D object recognition and reconstruction in unordered datasets", In: Fifth International Conference on 3-D Digital Imaging and Modeling (3DIM'05), Ottawa, Canada, 2005, pp. 56-63. https://doi.org/10.1109/3DIM.2005.81

[4] Micheletti, N., Chandler, J. H., Lane, S. N. "Structure from motion (SFM) photogrammetry", In: Clarke, L. E., Nield, J. M. (eds.) Geomorphological Techniques, British Society for Geomorphology, London, UK, 2015.

[5] Westoby, M. J., Brasington, J., Glasser, N. F., Hambrey, M. J., Reynolds, J. M. "Structure-from-Motion' photogrammetry: A lowcost, effective tool for geoscience applications", Geomorphology, 179, pp. 300-314, 2012.

https://doi.org/10.1016/j.geomorph.2012.08.021

[6] Cucchiaro, S., Maset, E., Fusiello, A., Cazorzi, F. "4d-SFM photogrammetry for monitoring sediment dynamics in a debris-flow catchment: software testing and results comparison", International Archives of the Photogrammetry, Remote Sensing and Spatial Information Sciences, 42(2), pp. 281-288, 2018. https://doi.org/10.5194/isprs-archives-XLII-2-281-2018
Using the several plug-in and other tools implemented in Rhinoceros software, it was possible to obtain a 3D model of the individual elements of the bridge. In addition, this latter task is useful and indispensable in structures where natural obstacles, such as vegetation or extrinsic artefacts to the bridge structure, do not allow an accurate and realistic reconstruction of the model under investigation.

The Hyperlink command, developed in Rhinoceros, allowed linking to an object other semantic information. This tool is particularly useful to program, manage and perform maintenance operations on structures belonging to cultural heritage, as shown in the case study of the masonry bridge. In addition, the modelling of individual elements in 3D not only allows a more efficient method for the three-dimensional representation of the bridge but also is a very useful tool for subsequent implementation in FEM (Finite Element Method) software to analyze the static and dynamic aspects of the structure.

[7] Brede, B., Lau, A., Bartholomeus, H. M., Kooistra, L. "Comparing RIEGL RiCOPTER UAV LiDAR Derived Canopy Height and DBH with Terrestrial LiDAR", Sensors, 17(10), Article number: 2371, 2017. https://doi.org/10.3390/s17102371

[8] Scaioni, M., Crippa, J., Corti, M., Barazzetti, L., Fugazza, D., Azzoni, R., Diolaiuti, G. A. "Technical aspects related to the application of SFM photogrammetry in high mountain", International Archives of the Photogrammetry, Remote Sensing and Spatial Information Sciences, 42(2), pp. 1029-1036, 2018. https://doi.org/10.5194/isprs-archives-XLII-2-1029-2018

[9] Bolognesi, M., Furini, A., Russo, V., Pellegrinelli, A., Russo, P. "Accuracy of cultural heritage 3D models by RPAS and terrestrial photogrammetry", International Archives of the Photogrammetry, Remote Sensing and Spatial Information Sciences, 40(5), pp. 113$119,2014$.

https://oi.org/10.5194/isprsarchives-XL-5-113-2014

[10] Russo, M., Manferdini, A. M. "Integration of image and range-based techniques for surveying complex architectures", ISPRS Annals of Photogrammetry, Remote Sensing and Spatial Information Sciences, 2(5), pp. 305-312, 2014.

https://doi.org/10.5194/isprsannals-II-5-305-2014

[11] Kouimtzoglou, T., Stathopoulou, E. K., Agrafiotis, P., Georgopoulos, A. "Image-Based 3d Reconstruction Data as AN Analysis and Documentation Tool for Architects: the Case of Plaka Bridge in Greece", International Archives of the Photogrammetry, Remote Sensing and Spatial Information Sciences, 42(2/W3), pp. 391-397, 2017.

https://doi.org/10.5194/isprs-archives-XLII-2-W3-391-2017 
[12] Hatzopoulos, J. N., Stefanakis, D., Georgopoulos, A., Tapinaki, S., Pantelis, V., Liritzis, I. "Use of various surveying technologies to $3 \mathrm{~d}$ digital mapping and modelling of cultural heritage structures for maintenance and restoration purposes: the tholos in Delphi, Greece", Mediterranean Archaeology and Archaeometry, 17(3), pp. 311-336, 2017.

https://doi.org/10.5281/zenodo.1048937

[13] Kaimaris, D., Karadedos, G., Georgiadis, C., Patias, P. "Locating and Mapping the Traces of the Covered Ancient Theater of Amphipolis (Eastern Macedonia, Greece)", Heritage, 1(2), pp. 306-319, 2018. https://doi.org/10.3390/heritage1020020

[14] Pepe, M., Costantino, D., Restuccia Garofalo, A. "An Efficient Pipeline to Obtain 3D Model for HBIM and Structural Analysis Purposes from 3D Point Clouds", Applied Sciences, 10(4), Article number: 1235, 2020. https://oi.org/10.3390/app10041235

[15] Belli, P. "Ponti in muratura di fine '800 nell'Italia meridionale" (Masonry bridges from the end of the 19th century in southern Italy), In: Proceedings of the 2nd National Conference on the History of Engineering, Naples, Italy, April, 7-9, 2008.

[16] Romano, A., Ochsendorf, J. A. "Circular, Pointed and BasketHandle Arches: A Comparison of Structural Behavior of Masonry Spans", In: Lourenço, P. B., Roca, P., Modena, C., Agrawal, S. (eds.) Structural Analysis of Historical Constructions, Macmillan Publishers India, Noida, India, 2006, pp. 1205-1212.

[17] Pepe, M. "Image-based methods for metric surveys of buildings using modern optical sensors and tools: from $2 \mathrm{D}$ approach to $3 \mathrm{D}$ and vice versa", International Journal of Civil Engineering and Technology (IJCIET), 9(9), pp. 729-745, 2018.

[18] Parrot "Anafi The 4K HDR Compact Drone for Your Aerial Shots" [online] Available at: https://www.parrot.com/us/drones/anafi [Accessed: 30 October 2019]

[19] Rabah, M., Basiouny, M., Ghanem, E., Elhadary, A. "Using RTK and VRS in direct geo-referencing of the UAV imagery", NRIAG Journal of Astronomy and Geophysics, 7(2), pp. 220-226, 2018. https://doi.org/10.1016/j.nrjag.2018.05.003
[20] Pepe, M., Fregonese, L., Crocetto, N. "Use of SFM-MVS Approach to Nadir and Oblique Images Generated throught Aerial Cameras to Build 2.5 D Map and 3D Models in Urban Areas", Geocarto International, 2019. https://doi.org/10.1080/10106049.2019.1700558

[21] Somogyi, Á., Fehér, K., Lovas, T., Halmos, B., Barsi, Á. "Analysis of Gothic Architectural Details by Spatial Object Reconstruction Techniques", Periodica Polytechnica Civil Engineering, 61(3), pp. 640-651, 2017. https://doi.org/10.3311/PPci.10418

[22] Kordić, B., Lužar-Oberiter, B., Pikelj, K., Matoš, B., Vlastelica, G. "Integration of Terrestrial Laser Scanning and UAS Photogrammetry in Geological Studies: Examples from Croatia", Periodica Polytechnica Civil Engineering, 63(4), pp. 989-1003, 2019. https://doi.org/10.3311/PPci.14499

[23] Agisoft "Agisoft Metashape User Manual, Professional Edition, Version 1.5", [pdf] 2019. Available at: https://www.agisoft.com/ pdf/metashape-pro_1_5_en.pdf [Accessed: 30 October 2019]

[24] Kraus, K. "Photogrammetry. Vol. 1: Fundamentals and Standard Processes", Dümmler, Bonn, Germany, 1994.

[25] Pepe, M., Fregonese, L., Scaioni, M. "Planning airborne photogrammetry and remote-sensing missions with modern platforms and sensors", European Journal of Remote Sensing, 51(1), pp. 412436, 2018. https://doi.org/10.1080/22797254.2018.1444945

[26] Federman, A., Santana Quintero, M., Kretz, S., Gregg, J., Lengies, M., Ouimet, C., Laliberte, J. "UAV photogrammetric workflows: A best practice guideline", International Archives of the Photogrammetry, Remote Sensing and Spatial Information Sciences, 42(2/W5), pp. 237-244, 2017. https://doi.org/10.5194/isprs-archives-XLII-2-W5-237-2017

[27] Rhinocentre "Rhinoceros 6.0" [online] Available at: https://www. rhinocentre.com/product/rhino-6-0-win/ [Accessed: 30 October 2019] 


\section{Appendix A}

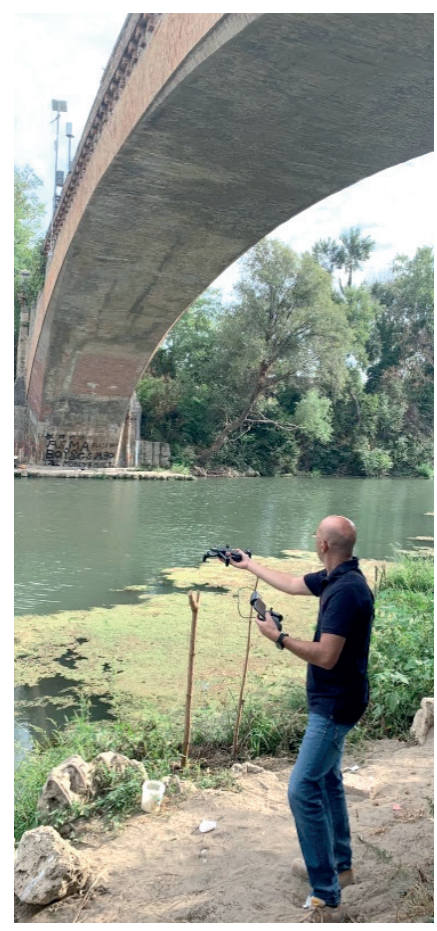

Fig. 12 Hand launch of the UAV under the bridge

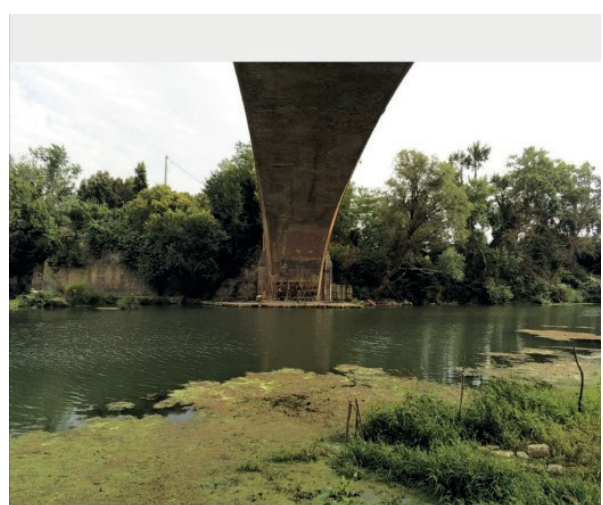

Visualizzare le foto >

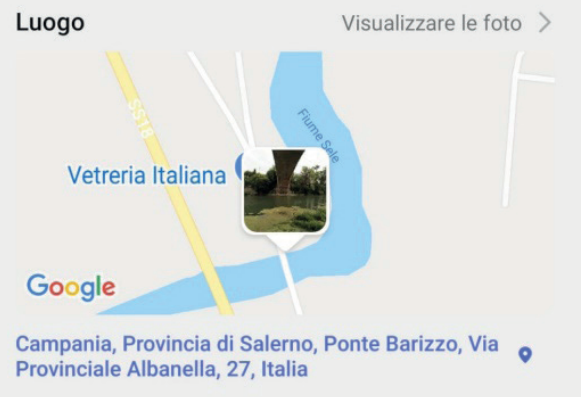

Fig. 13 Image acquired by Parrot UAS and, by geotagging, the picture is visualised in Google Maps

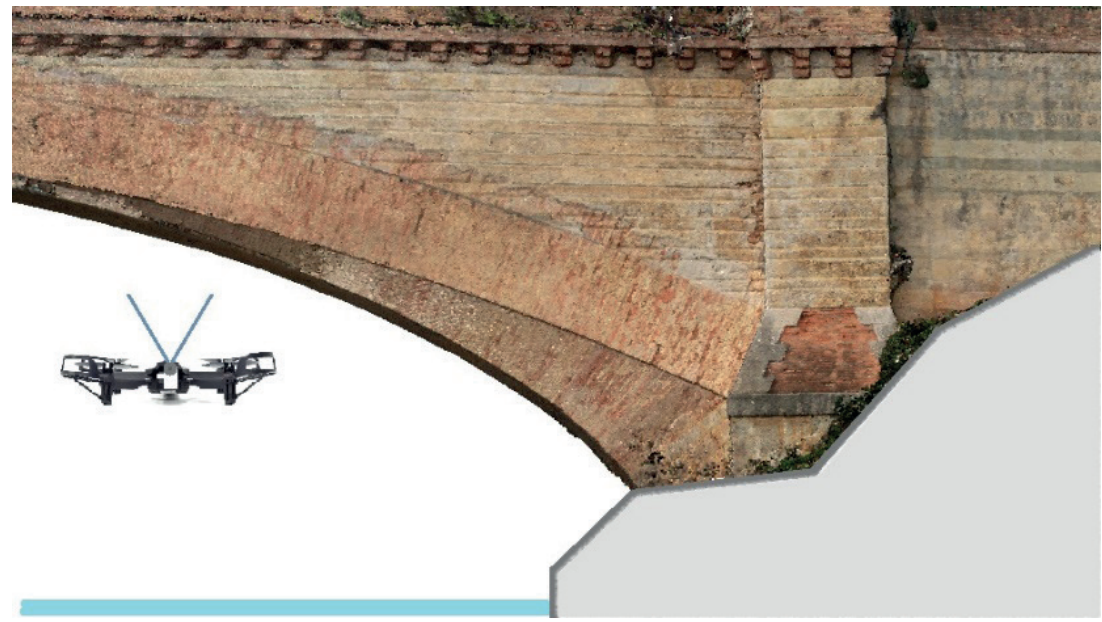

Fig. 15 Sketch of image capture mode in the direction of the zenith

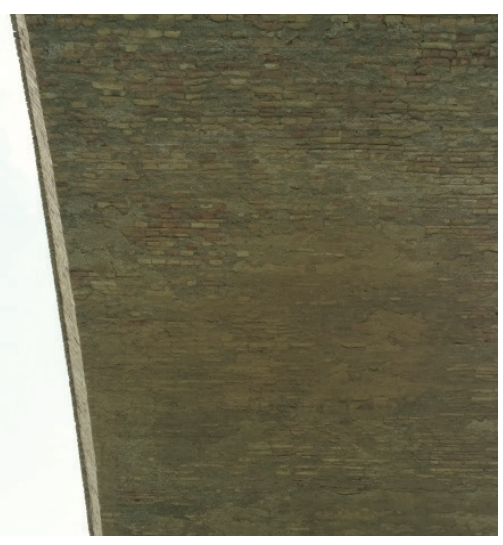

Fig. 14 Image acquired in the direction of the zenith

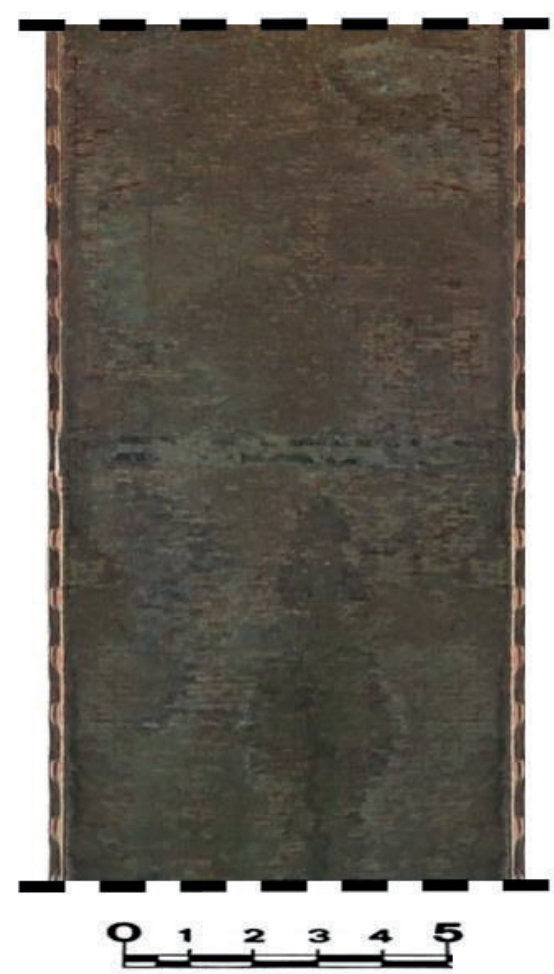

Fig. 16 Orthophoto Down-view of the middle part of the bridge 